\title{
Tocilizumab in Treatment of Severe COVID-19 Patients: A Systematic Review and Meta-Analysis of Cohort Studies
}

\author{
Mohamed Elsokary ${ }^{1}$, Hozaifa Elsawah ${ }^{1}$, Ahmed Elshafie ${ }^{2}$, and Mahmoud Abdallah ${ }^{3}$ \\ ${ }^{1}$ Alexandria University High Institute of Public Health \\ ${ }^{2}$ Shebin Elkom Teaching Hospital \\ ${ }^{3}$ University of Sadat City
}

September 21, 2020

\begin{abstract}
Background: There is an urgent need to improve health outcomes among severe COVID-19 patients. Therefore, this study aimed to demystify efficacy and safety of tocilizumab among those patients based on the best available evidence. Methods: Nine electronic databases were searched for relevant studies by three authors in July using search terms "tocilizumab", "COVID", "SARS". The last date checked was 26 August, 2020. Eligible studies were assessed through an inclusion criterion focused on cohort studies and severe COVID-19 adult patients. Any disagreement between the authors was resolved by discussions. Primary outcomes were mortality and clinical improvement, while secondary outcomes were tocilizumab-related adverse effects. Results: Tocilizumab reduced the mortality rate by $41 \%, 40 \%, 28 \%$, and $37 \%$ in $7,14,21$, and 28 days. There was neither statistically significant difference between the tocilizumab group and the control group in the clinical improvement (pooled risk ratio1.21 $[0.89,1.64])$ nor in respiratory support change. There were no difference between the two groups in the occurrence of serious adverse events (pooled risk difference $0.00[-0.02,0.03]$ ), bacteremia (pooled risk ratio 1.25 [0.80, 1.97]), elevation of liver functions (pooled risk difference $-0.00[-0.03,0.02]$ ) and infusion related reactions (pooled risk difference 0.01 [-0.02, 0.03]). However, the occurrence of neutropenia (pooled risk ratio $9.23[1.06,80.24]$ ) and new infections (pooled risk difference 0.07 $[0.00,0.14])$ were more likely in the tocilizumab group. Conclusion: Because of the reported adverse effects of tocilizumab in treating severe COVID-19 patients, the use of that biological agent should be limited to selected patients based on the best available evidence.
\end{abstract}

Tocilizumab in Treatment of Severe COVID-19 Patients: A Systematic Review and MetaAnalysis of Cohort Studies

Short running title: Tocilizumab for severe Covid-19

Mohamed Ahmed Elsokary PharmD ${ }^{1}$, Hozaifa Khalil Elsawah PharmD, MSc ${ }^{1}$, Ahmed Hanei Elshafie $\mathrm{MSc}^{2}$, Mahmoud S. Abdallah $\mathrm{PhD}^{3}$

${ }^{1}$ Department of Biostatistics, High Institute of Public Health, Alexandria University, Alexandria, Egypt

${ }^{2}$ Department of Neuropsychiatry, Shebin Elkom Teaching Hospital, Shebin Elkom, Egypt

${ }^{3}$ Department of Clinical Pharmacy, Faculty of Pharmacy, University of Sadat City (USC), Sadat City, Menoufia, 32897, Egypt

Correspondence:

Mohamed Ahmed Elsokary, Alexandria University, Alexandria, Egypt.

ORCID: https://orcid.org/0000-0002-9832-5086 
Email: sokkary.mhmed@gmail.com

Present address

Mohamed Ahmed Elsokary, Quesna, Monofiya, 32631, Egypt

\section{Summary:}

Background: There is an urgent need to improve health outcomes among severe COVID-19 patients. Therefore, this study aimed to demystify efficacy and safety of tocilizumab among those patients based on the best available evidence.

Methods: Nine electronic databases were searched for relevant studies by three authors in July using search terms "tocilizumab", "COVID", "SARS". The last date checked was 26 August, 2020. Eligible studies were assessed through an inclusion criterion focused on cohort studies and severe COVID-19 adult patients. Any disagreement between the authors was resolved by discussions. Primary outcomes were mortality and clinical improvement, while secondary outcomes were tocilizumab-related adverse effects.

Results: Tocilizumab reduced the mortality rate by $41 \%, 40 \%, 28 \%$, and $37 \%$ in $7,14,21$, and 28 days. There was neither statistically significant difference between the tocilizumab group and the control group in the clinical improvement (pooled risk ratio1.21 [0.89, 1.64]) nor in respiratory support change. There were no difference between the two groups in the occurrence of serious adverse events (pooled risk difference $0.00[-0.02,0.03]$ ), bacteremia (pooled risk ratio $1.25[0.80,1.97]$ ), elevation of liver functions (pooled risk difference $-0.00[-0.03,0.02]$ ) and infusion related reactions (pooled risk difference $0.01[-0.02,0.03]$ ). However, the occurrence of neutropenia (pooled risk ratio $9.23[1.06,80.24]$ ) and new infections (pooled risk difference $0.07[0.00,0.14])$ were more likely in the tocilizumab group.

Conclusion: Because of the reported adverse effects of tocilizumab in treating severe COVID-19 patients, the use of that biological agent should be limited to selected patients based on the best available evidence.

Key Words : Tocilizumab; COVID-19; SARS COV 2; Systematic Review; Meta-Analysis; Cohort studies

\section{Introduction}

SARS COV 2 is a member of Corona virus family, which was first recognized in December 2019 in the Chinese city of Wuhan and caused what is known nowadays as the COVID 19 pandemic (Cascella, Rajnik, Cuomo, Dulebohn, \& Di Napoli, 2020). The virus is believed to be transmitted by droplets and aerosols causing various clinical conditions ranging from mild flu-like symptoms up to very severe conditions such as acute respiratory distress syndrome (ARDS) (Jayaweera, Perera, Gunawardana, \& Manatunge, 2020). Expected death rate among severe ARDS cases is estimated to be up to $62 \%$ (Huang et al., 2020) which is assumed to be a result of what is known as a cytokine storm (Ulhaq \& Soraya, 2020). Global attention was therefore paid to the emerging pandemic, in particular the development of new vaccines (Chugh, 2020).

Expanding evidence shows that inflammatory mediators, such as interferons, interleukins, chemokines, tumor-necrotic factors, etc, may be activated by severe COVID-19 condition in the case of a hyperactive immunological response (Yuki, Fujiogi, \& Koutsogiannaki, 2020). These mediators are inherent in the innate immune system that attacks foreign infectious agents. However, in very severe conditions it triggers a life-threatening immunological reaction, including a massive release of cytokines which is supposed to cause a cytokine storm (Ye, Wang, \& Mao, 2020). This cytokine storm is followed by the immune system attacking the body, which in turn causes ARDS and multiple organ failure (Siracusano, Pastori, \& Lopalco, 2020; Z. $\mathrm{Xu}$ et al., 2020). Recent studies have demonstrated that patients with severe COVID-19 have elevated serum levels of pro-inflammatory cytokines, including interleukins-6 (IL-6) (Huang et al., 2020; Wang et al., 2007). 
Based on that hypothesis, modulating the hyperinflammatory status associated with COVID-19 has been targeted by some drugs in clinical trials including dexamethasone that showed promising improvement in RECOVERY trial (Horby et al., 2020), while the others failed to show significant improvement such as hydroxychloroquine in a recent meta-analysis of clinical trials (Elsawah, Elsokary, Elrazzaz, \& ElShafey, 2020).

Tocilizumab is a humanized anti-IL-6 receptor IgG1 monoclonal antibody used for the treatment of rheumatoid arthritis and other chronic inflammatory diseases (Arnaldez et al., 2020). By blocking of IL-6- receptor binding, tocilizumab inhibits signal transduction mediated by IL-6 (Nishimoto \& Kishimoto, 2008). Chi Zhang in China first highlighted the cytokine storm hypothesis in severe COVID-19 and the potential impact of tocilizumab against IL-6 and recommended further evidence-based studies to co-relate the potential benefits (Chi, Zhao, Jia-Wen, Hong, \& Gui-Qiang, 2020). Furthermore, Xiaoling Xu et al ., claimed effective clinical improvement and successful repression of clinical deterioration in severe COVID-19 patients in their single arm observational study (X. Xu et al., 2020).

Another observational case control study conducted in Italy by Ruggero Capra et al ., showed promising data showing a significant reduction in the mortality rates in the tocilizumab group compared to the control group (Capra et al., 2020). On the other hand, a phase 3 clinical trial conducted by Roche failed to show beneficial outcome regarding clinical status (Hoffmann-La Roche, 2020). Meanwhile, cohort studies were conducted to investigate different outcomes including the overall improvement, length of stay, mortality rates and safety profile (Campochiaro et al., 2020; Colaneri et al., 2020; Guaraldi et al., 2020). In view of the conflicting results, we aimed to systemically review these cohort studies and conduct an updated meta-analysis to provide the best evidence. Our stated objectives are to disclose possible protective effects of tocilizumab from the need for mechanical ventilation and mortality in severe COVID-19 patients, to pool any clinical improvement associated with tocilizumab use, and to summarize the adverse effects reported with tocilizumab use in severe COVID-19 patients.

\section{Methods:}

The Preferred Reporting Items for Systematic Reviews and Meta-Analyses (PRISMA) Checklist was followed to improve reporting this meta-analysis

\section{Protocol registration:}

The protocol was registered in the International Prospective Reregister of Systematic Review with registration number CRD42020204233 in August 2020.

1.

\section{Criteria for considering studies for this review}

\section{Types of the studies:}

Cohort studies either prospective or retrospective comparing the use of tocilizumab with placebo in presence of standard of care that was given to all patients according to need. Case-control studies were excluded.

\section{Types of participant:}

Patients with severe COVID-19 patients defined as those with at least reduced oxygen saturation $(<94 \%)$ or required ICU admission and patients with PCR-confirmed COVID-19. Evidence of hyperinflammatory status is not necessary but will be highlighted. 


\section{Types of interventions:}

Tocilizumab intravenous (IV) with dose 400 to $800 \mathrm{mg}$ once and could be repeated for another dose.

\section{Types of outcome measures}

Efficacy and adverse effects that were reported up to 28 days follow-up period

\section{Primary outcomes}

Mortality during 28 days-follow-up, need for mechanical ventilation, and clinical improvement including hospital discharge and change in respiratory support.

\section{Secondary outcomes}

Serious adverse effects and occurrence of new infections

\section{Additional outcomes}

Incidence of elevation in liver enzymes, infusion-related reaction, neutropenia, and bacteremia.

1.

\section{Search methods for identification of studies}

\section{Information sources:}

The following databases have been checked from the first of July 2020 and continued through August to identify relevant studies: ClinicalTrial.gov, ProQuest, PubMed, Embase, Cochrane, Google Scholar, Science direct, Chinese Clinical Trial Registry (ChiCTR), and medRxiv.

The following search terms were used: "actemra", "tocilizumab", "SARS" and "COVID." In addition, a deep manual search through checking references in bibliography of original articles and relevant reviews was performed.

\section{Study selection:}

Studies were selected based on PRISMA flow diagram (Fig. 1 ). Two authors (HKE and MAE) independently selected potentially eligible studies from screened ones based on the title and/or abstract. The full text has been presented in order to review the articles screened for inclusion criteria. The reasons for excluded studies were discussed and any disagreements were resolved through discussion. A third author (MS) revised the process of the study selection and eligibility assessment.

\section{Data collection and analysis:}

Data was extracted using a predefined extraction form. The form included:

Study characteristics: title, authors, year of publication, country, and journal name.

Study design: type of the study design, hospital name, time of interventions, time of follow-up, and other settings

Population: age, sex, baseline clinical factors, disease severity definition, inflammation status, inclusion and exclusion criteria, and the disease onset

Intervention: Tocilizumab dose, route of administration, and duration.

Comparator: Any other treatment or care that was given to the patients

Outcomes: All outcomes either efficacy or safety-related were extracted extensively including numbers or data presented with graphs that were transformed with a specific software; Get Data Graph Digitizer 2.26 


\section{Assessment of risk of bias in included studies:}

The methodological quality of selected cohort studies were assessed based on the basis of " risk of bias in nonrandomized studies of interventions" (ROBINS-I) (Sterne et al., 2016); a tool provides a more comprehensive framework for identifying potential sources of bias (Losilla, Oliveras, Marin-Garcia, \& Vives, 2018). The following points were scored as low, moderate, serious, critical or no information (where 'low' indicated that the study was less risk to bias and thus best quality), and were reported in a "Risk of bias figure": bias due to confounding, bias in selection of participants into the study, bias in classification of interventions, bias due to deviations from intended interventions, bias due to missing data, bias in measurement of outcomes, and bias in selection of the reported result.

A consensus criteria in the present meta-analysis for bias judgment included that a study was judged at low risk of bias if all key domains were judged at low risk of bias, a study was judged at high risk of bias if two or more key domains were judged at high risk of bias, otherwise the study was judged at unclear risk of bias.

Studies of low quality were not excluded, instead they were involved in data synthesis after performing sensitivity analysis.

\section{Synthesis of the quantitative results}

Measures of treatment effect: risk ratios (RR) with 95\%confidence intervals (CI) was used to analyze dichotomous data. None of our included studies reported continuous data.

Data synthesis: The overall treatment effect was estimated by the pooled RR with $95 \%$ CI by RevMan version 5.4 using a fixed-effect model (Mantel-Haenszel). A random effects model was used in cases of significant heterogeneity.

Assessment of heterogeneity: Chi-square test of heterogeneity and the $\mathrm{I}^{2}$ statistic of heterogeneity were used to assess effects heterogeneity. For Chi-square test, the data study findings were considered to be heterogeneous if $P$-value was [?] 0.05 . When a significant heterogeneity occurred, the differences were explained as they related to types of participants and study design.

Sensitivity analysis: Sensitivity analysis was conducted for only those cohort studies assessed as having a low overall risk of bias based on our consensus criteria in key domains.

Publication bias: The publication bias was assessed by using funnel plots when there were more than 10 studies reporting the same effect measure of an outcome.

\section{Results:}

\section{Study selection:}

PRISMA flow diagram in figure (1) shows that 826 studies were identified after removal of duplications, then 789 irrelevant studies were excluded after titles and abstracts screening. Finally, 37 full articles were assessed for eligibility based on the prespecified inclusion criteria. The remaining 6 studies were included in the systematic review and meta-analysis. Some studies were excluded because they had case-control designs (Klopfenstein et al., 2020; Rojas-Marte et al., 2020; Rossotti et al., 2020), while the others were preprint (Rossi et al., 2020) that may be included in the next updates.

\section{Study characteristics:}

The population of the included studies had a severe COVID-19 infection. The disease severity criteria are well defined in each study with minor variations. A prespecified severity criteria were found in four studies which include impaired oxygenation in common (Campochiaro et al., 2020; Colaneri et al., 2020; Guaraldi et 
al., 2020; Kewan et al., 2020) in addition to typical radiological findings (Campochiaro et al., 2020; Guaraldi et al., 2020; Kewan et al., 2020), while the other two studies focused on critical care patients (Ip et al., 2020) and those who required mechanical ventilation (Somers, Eschenauer, Troost, Golob, Gandhi, Wang, Zhou, Petty, Baang, Dillman, et al., 2020). Evidence of hyperinflammation was required for tocilizumab indication in three studies (Campochiaro et al., 2020; Ip et al., 2020; Kewan et al., 2020)

TCZ regimen was one or two doses, each was 400-800 mg.

Routine treatment was given to all patients according to needs and varied widely among the studies. It included: supportive care, symptomatic treatment, steroids, antibiotics, hydroxychloroquine, anticoagulants and antivirals (Table 1 ).

Median onset of COVID-19 symptoms before starting the treatment in tocilizumab and control group respectively was 11 and 9 days in Campochiaro et al ., study (Campochiaro et al., 2020), 4 and 5 days in Guaraldi et al ., study (Guaraldi et al., 2020), and 5 days in Andrew et al ., study (Ip et al., 2020).

\section{Risk of bias within studies:}

The risk of bias is summarized in Table (2). As an observational study, cohort design is highly vulnerable to confounding, therefore it is necessary to show comparable groups in baseline factors that can predict the outcomes. Two studies did not give enough data about the significance of these differences (Colaneri et al., 2020; Kewan et al., 2020), while another two showed serious bias risk (Guaraldi et al., 2020; Ip et al., 2020). Selection bias and misclassification bias were low in all studies. Bias due to deviations from intended interventions was moderate in three studies because of unequal background treatment including steroids between the groups (Guaraldi et al., 2020; Ip et al., 2020; Kewan et al., 2020) . Bias in measurement of outcomes was moderate in all studies because of the retrospective design-related recall bias risk. Bias in selection of the reported result was moderate in two studies (Kewan et al., 2020; Somers, Eschenauer, Troost, Golob, Gandhi, Wang, Zhou, Petty, Baang, \& Dillman, 2020) as they did not report data about many clinical outcomes and adverse effects associated with tocilizumab use.

\section{Synthesis of results:}

Included studies recruited 1473 patients with confirmed severe COVID-19. Of them, 472 patients received tocilizumab while 1001 patients served as a control. Male participants were predominant in all the studies. The male sex OR in TCZ treated group relative to control pooled from the studies using fixed effect model was $1.59[1.24,2.04]$.

\section{The main outcomes:}

1. As demonstrated in Fig. 2, the pooled mortality risk from the included six studies was significantly reduced by $41 \%$ in 7 days, $40 \%$ in 14 days, $28 \%$ in 21 days, and $37 \%$ in 28 days in the tocilizumab treated group, compared to the control group using the fixed-effect model. Heterogeneity was not significant $(\mathrm{P}>0.05)$.

2. In Fig. 3 , the clinical improvement was not statistically significant between the two groups. RR pooled from two studies (Campochiaro et al., 2020; Kewan et al., 2020) using fixed effect model was $1.21[0.89,1.64]$. Heterogeneity was not significant $(P=0.72)$.

3. There was no statistically significant difference between the two groups in the change in respiratory support. RR of improvement and worsening in respiratory support pooled from two studies (Campochiaro et al., 2020; Kewan et al., 2020) using fixed effect model was $1.20[0.91,1.57]$ and $0.52[0.26$, 1.04], respectively. Heterogeneity was not significant $(\mathrm{P}>0.05)$.

4. 


\section{Additional outcomes:}

1. In Fig. 4, the occurrence of serious adverse events was not significant between the two groups. RD pooled from three studies (Campochiaro et al., 2020; Colaneri et al., 2020; Guaraldi et al., 2020) using fixed effect model was $0.00[-0.02,0.03]$. Heterogeneity was not significant $\left(\chi^{2}=0.32, P=0.85\right)$.

2. The occurrence of bacteremia was not significant between the two groups. RR pooled from three studies (Campochiaro et al., 2020; Guaraldi et al., 2020; Ip et al., 2020) using fixed effect model was $1.25[0.80,1.97]$. Heterogeneity was not significant $\left(\chi^{2}=0.16, P=0.92\right)$

3. The elevation of liver functions was not significant between the two groups. RD pooled from three studies (Campochiaro et al., 2020; Colaneri et al., 2020; Guaraldi et al., 2020) using fixed effect model was $-0.00[-0.03,0.02]$. Heterogeneity was not significant $\left(\chi^{2}=0.24, P=0.89\right)$.

4. Neutropenia was 9.23 more likely to be occurred in tocilizumab treated group than control group. RR pooled from two studies (Campochiaro et al., 2020; Guaraldi et al., 2020)using fixed effect model was $9.23[1.06,80.24]$. Heterogeneity was not significant $\left(\chi^{2}=0.08, P=0.77\right)$

5. The occurrence of new infections was significant between the two groups. RD pooled from six studies (Campochiaro et al., 2020; Colaneri et al., 2020; Guaraldi et al., 2020; Ip et al., 2020; Kewan et al., 2020; Somers, Eschenauer, Troost, Golob, Gandhi, Wang, Zhou, Petty, Baang, \& Dillman, 2020) using fixed and random effect model was $0.09[0.05,0.12]$ and $0.07[0.00,0.14]$ respectively. Heterogeneity was significant $\left(\chi^{2}=18.73, P=0.002\right)$.

6. The occurrence of infusion related reaction was not significant between the two groups. RD pooled from two studies (Campochiaro et al., 2020; Guaraldi et al., 2020) using Fixed effect model was 0.01 $[-0.02,0.03]$. Heterogeneity was not significant $\left(\chi^{2}=0.12, P=0.73\right)$.

7.

\section{Sensitivity analysis:}

Based on our assessment of risk of bias, the only two studies showed serious risk of confounding bias, Guaraldi G. et al., and Ip A.et al., were excluded. The same analysis of the combined outcomes was done, and there was no significant difference between the two analyses.

\section{Discussion}

To the best of our knowledge, this study is the first meta-analysis built only on the most recent cohort studies to investigate the safety and efficacy of tocilizumab in severe COVID-19. The study searched for the best available evidence evaluating the role of tocilizumab in patients with severe COVID-19 and provided moderate to high quality retrospective cohort studies including 1473 patients in 6 studies. Tocilizumab succeeded in reducing the mortality rate significantly at all-time points; 7, 14, 21, 28 days pooled from all the included studies. Moreover, heterogeneity among the study findings was not significant ensuring that the study findings are consistent. The preventive effect tocilizumab from all-cause mortality was confounded by unequal distribution of some baseline factors, therefore sensitivity analysis was performed after removing the most confounded studies. Repeating the analysis after exclusion of the only two studies with serious risk of confounding bias ensured that the conclusion is robust through finding a little or not at all change in the results after exclusion of the two studies.

Tocilizumab was not superior to the control group regarding improvement in the respiratory supportive level and clinical improvement. This may be due to the low number of patients and studies included in the analysis to estimate pooled RR of either clinical improvement or improvement in the respiratory support level. 
In contrast to our results, a recently published meta-analysis that was conducted by Lan et al., (Lan et al., 2020) failed to show significant reduction in all-cause mortality associated with tocilizumab. These results could be explained by the difference in the included studies, where they pooled case-control studies and pre-print studies. Furthermore, the safety of tocilizumab was not investigated.

Regarding the safety of the tocilizumab, the present meta-analysis reported that the incidence of neutropenia and new infections were the only statistically significant parameters in the tocilizumab group. The overall safety outcome favored the control group, although it did not affect the mortality rate because the majority of the reported cases were mild.

Many side effects of tocilizumab have been reported since it has been approved for us in different indications. It includes neutropenia, thrombocytopenia, increased liver enzymes, hypersensitivity, hyperlipidemia, infection, and injection site reactions..etc (Bannwarth \& Richez, 2011).

A recent phase 3 clinical trial conducted by Roche was published in August 2020 and provided disappointing results regarding the efficacy of the tocilizumab in the treatment of severe COVID-19 patients (Roche, 2020). This comes congruent with the results provided by our systematic review and metanalysis.

We recommend further randomized clinical trials including larger sample size to be conducted in different stages of the disease, and further studies to clearly identify the exact pathogenesis behind the COVID-19 cytokine storm.

The current evidence of using tocilizumab in severe COVID-19 was based on having a hyper inflammatory conditions (Campochiaro et al., 2020; Ip et al., 2020; Kewan et al., 2020) rather than using H-score to predict cytokines storm which is not validated in COVID-19 patients (Leverenz \& Tarrant, 2020). Actually, it was validated to predict hemophagocytic lymphohistiocytosis (HLH) in rheumatic patients (Fardet et al., 2014). Patients' stratification and well-defined criteria for hyper inflammation-related COVID-19 should be addressed in the future studies to maximize the tocilizumab benefits.

\section{Limitation:}

There are some limitations in this study. Firstly, the patients could not be stratified by baseline mechanical ventilation assuming that tocilizumab efficacy is the same because of the low number of the included studies. Secondly, cohort studies are highly susceptible for confounding that may affect the internal validity of the treatment-effect relationship. Thirdly, immunomodulators such as steroids were given beyond tocilizumab in three studies (Guaraldi et al., 2020; Ip et al., 2020; Kewan et al., 2020) which could have an effect change. Finally, some other outcomes could not be combined due to difference in the follow-up time such as a rate of mechanical ventilation during 14 (Guaraldi et al., 2020), 21 (Kewan et al., 2020), and 28 days (Campochiaro et al., 2020).

\section{Conclusion:}

Tocilizumab administration in subjects with severe COVID-19 infection reduced the mortality rate in all time points, however, the incidence of adverse effect was higher. Therefore, patients with severe COVID19 specially those with hyperinflammation may benefit from tocilizumab use taking into consideration the adverse effects of the biological drug. However, the limitations of the study would encourage researchers to conduct further investigations with randomized clinical trials.

Ethics Statement: The authors confirm that the ethical policies of the journal, as noted on the journal's author guidelines page, have been adhered to. No ethical approval was required as this is a review article with no original research data 


\section{Conflict of interest:}

On behalf of all authors, the corresponding author states that there is no conflict of interest.

Funding source: None

Data Availability Statement : Data sharing not applicable to this article as no datasets were generated or analysed during the current study

\section{References:}

Arnaldez, F. I., O’Day, S. J., Drake, C. G., Fox, B. A., Fu, B., Urba, W. J., . . . Ascierto, P. A. (2020). The Society for Immunotherapy of Cancer perspective on regulation of interleukin-6 signaling in COVID-19-related systemic inflammatory response. Journal for immunotherapy of cancer, 8 (1), e000930. doi:10.1136/jitc-2020-000930

Bannwarth, B., \& Richez, C. (2011). Clinical safety of tocilizumab in rheumatoid arthritis. Expert opinion on drug safety, 10 (1), 123-131.

Campochiaro, C., Della-Torre, E., Cavalli, G., De Luca, G., Ripa, M., Boffini, N., . . . Ruggeri, A. (2020). Efficacy and safety of tocilizumab in severe COVID-19 patients: a single-centre retrospective cohort study. European journal of internal medicine .

Capra, R., De Rossi, N., Mattioli, F., Romanelli, G., Scarpazza, C., Sormani, M. P., \& Cossi, S. (2020). Impact of low dose tocilizumab on mortality rate in patients with COVID-19 related pneumonia.European journal of internal medicine .

Cascella, M., Rajnik, M., Cuomo, A., Dulebohn, S. C., \& Di Napoli, R. (2020). Features, evaluation and treatment coronavirus (COVID-19) Statpearls [internet] : StatPearls Publishing.

Chi, Z., Zhao, W., Jia-Wen, L., Hong, Z., \& Gui-Qiang, W. (2020). Cytokine release syndrome in severe COVID-19: interleukin-6 receptor antagonist tocilizumab may be the key to reduce mortality.International journal of antimicrobial agents, 55 (5), 105954.

Chugh, T. (2020). Timelines of COVID-19 Vaccines. Current Medicine Research and Practice .

Colaneri, M., Bogliolo, L., Valsecchi, P., Sacchi, P., Zuccaro, V., Brandolino, F., . . . Bruno, R. (2020). Tocilizumab for Treatment of Severe COVID-19 Patients: Preliminary Results from SMAtteo COvid19 REgistry (SMACORE). Microorganisms, 8 (5), 695.

Elsawah, H. K., Elsokary, M. A., Elrazzaz, M. G., \& ElShafey, A. H. (2020). Hydroxychloroquine for treatment of non-severe COVID-19 patients; systematic review and meta-analysis of controlled clinical trials. Journal of Medical Virology .

Fardet, L., Galicier, L., Lambotte, O., Marzac, C., Aumont, C., Chahwan, D., . . . Hejblum, G. (2014). Development and validation of the HScore, a score for the diagnosis of reactive hemophagocytic syndrome.Arthritis $\& 3$ Rheumatology, 66 (9), 2613-2620.

Guaraldi, G., Meschiari, M., Cozzi-Lepri, A., Milic, J., Tonelli, R., Menozzi, M., . . . Borghi, V. (2020). Tocilizumab in patients with severe COVID-19: a retrospective cohort study. The Lancet Rheumatology .

Hoffmann-La Roche, A. (2020). Study to Evaluate the Safety and Efficacy of Tocilizumab in Patients With Severe COVID-19 Pneumonia (COVACTA) . Retrieved from https://www.roche.com/dam/jcr:6d8de90d2e31-43c8-b4e1-0a24a2675015/en/29072020-mr-covacta.pdf

Horby, P., Lim, W. S., Emberson, J., Mafham, M., Bell, J., Linsell, L., . . . Elmahi, E. (2020). Effect of dexamethasone in hospitalized patients with COVID-19: preliminary report. medRxiv . 
Huang, C., Wang, Y., Li, X., Ren, L., Zhao, J., Hu, Y., . . . Gu, X. (2020). Clinical features of patients infected with 2019 novel coronavirus in Wuhan, China. The lancet, 395 (10223), 497-506.

Ip, A., Berry, D. A., Hansen, E., Goy, A. H., Pecora, A. L., Sinclaire, B. A., . . . Berry, N. S. (2020). Hydroxychloroquine and Tocilizumab Therapy in COVID-19 Patients-An Observational Study. medRxiv .

Jayaweera, M., Perera, H., Gunawardana, B., \& Manatunge, J. (2020). Transmission of COVID-19 virus by droplets and aerosols: A critical review on the unresolved dichotomy. Environmental Research, 109819.

Kewan, T., Covut, F., Al-Jaghbeer, M. J., Rose, L., Gopalakrishna, K., \& Akbik, B. (2020). Tocilizumab for treatment of patients with severe COVID-19: A retrospective cohort study. EClinicalMedicine, 100418.

Klopfenstein, T., Zayet, S., Lohse, A., Balblanc, J.-C., Badie, J., Royer, P.-Y., . . . Bozgan, A.-M. (2020). Tocilizumab therapy reduced intensive care unit admissions and/or mortality in COVID-19 patients. Médecine et Maladies Infectieuses .

Lan, S.-H., Lai, C.-C., Huang, H.-T., Chang, S.-P., Lu, L.-C., \& Hsueh, P.-R. (2020). Tocilizumab for severe COVID-19: a systematic review and meta-analysis. International journal of antimicrobial agents , 106103.

Leverenz, D. L., \& Tarrant, T. K. (2020). Is the HScore useful in COVID-19? The lancet, 395 (10236), e83.

Losilla, J.-M., Oliveras, I., Marin-Garcia, J. A., \& Vives, J. (2018). Three risk of bias tools lead to opposite conclusions in observational research synthesis. Journal of clinical epidemiology, 101 , 61-72.

Nishimoto, N., \& Kishimoto, T. (2008). Humanized antihuman IL-6 receptor antibody, tocilizumab Therapeutic Antibodies (pp. 151-160): Springer.

Roche, H.-L. (2020). A Study to Evaluate the Safety and Efficacy of Tocilizumab in Patients With Severe COVID-19 Pneumonia (COVACTA) . Retrieved from https://www.roche.com/dam/jcr:6d8de90d-2e3143c8-b4e1-0a24a2675015/en/29072020-mr-covacta.pdf

Rojas-Marte, G., Khalid, M., Mukhtar, O., Hashmi, A. T., Waheed, M. A., Ehrlich, S., . . Malyshev, Y. (2020). Outcomes in patients with severe COVID-19 disease treated with tocilizumab: a case-controlled study.QJM: An International Journal of Medicine, 113 (8), 546-550.

Rossi, B., Nguyen, L. S., Zimmermann, P., Boucenna, F., Baucher, L., Dubret, L., . . Salem, J.-E. (2020). Effect of tocilizumab in hospitalized patients with severe pneumonia COVID-19: a cohort study.medRxiv .

Rossotti, R., Travi, G., Ughi, N., Corradin, M., Baiguera, C., Fumagalli, R., . . Bellone, A. (2020). Safety and efficacy of anti-il6-receptor tocilizumab use in severe and critical patients affected by coronavirus disease 2019: A comparative analysis. Journal of infection .

Siracusano, G., Pastori, C., \& Lopalco, L. (2020). Humoral Immune Responses in COVID-19 Patients: A Window on the State of the Art.Frontiers in Immunology, 11 .

Somers, E. C., Eschenauer, G. A., Troost, J. P., Golob, J. L., Gandhi, T. N., Wang, L., . . Dillman, N. O. (2020). Tocilizumab for treatment of mechanically ventilated patients with COVID-19. medRxiv .

Somers, E. C., Eschenauer, G. A., Troost, J. P., Golob, J. L., Gandhi, T. N., Wang, L., . . Pogue, J. M. (2020). Tocilizumab for treatment of mechanically ventilated patients with COVID-19. Clinical Infectious Diseases . doi:10.1093/cid/ciaa954

Sterne, J. A., Hernán, M. A., Reeves, B. C., Savović, J., Berkman, N. D., Viswanathan, M., . . . Boutron, I. (2016). ROBINS-I: a tool for assessing risk of bias in non-randomised studies of interventions.bmj, 355 .

Ulhaq, Z. S., \& Soraya, G. V. (2020). Interleukin-6 as a potential biomarker of COVID-19 progression. Médecine et Maladies Infectieuses .

Wang, W., Ye, L., Ye, L., Li, B., Gao, B., Zeng, Y., . . . Wu, Z. (2007). Up-regulation of IL-6 and TNF- $\alpha$ induced by SARS-coronavirus spike protein in murine macrophages via NF- $x \mathrm{~B}$ pathway. Virus research, 128 
$(1-2), 1-8$.

Xu, X., Han, M., Li, T., Sun, W., Wang, D., Fu, B., . . Li, X. (2020). Effective treatment of severe COVID19 patients with tocilizumab.Proceedings of the National Academy of Sciences, 117 (20), 10970-10975.

Xu, Z., Shi, L., Wang, Y., Zhang, J., Huang, L., Zhang, C., . . . Zhu, L. (2020). Pathological findings of COVID-19 associated with acute respiratory distress syndrome. The Lancet Respiratory Medicine, 8 (4), 420-422.

Ye, Q., Wang, B., \& Mao, J. (2020). The pathogenesis and treatment of theCytokine Storm'in COVID-19. Journal of infection, 80 (6), 607-613.

Yuki, K., Fujiogi, M., \& Koutsogiannaki, S. (2020). COVID-19 pathophysiology: A review. Clinical immunology , 108427.

Table (1): Characteristics of the included studies:

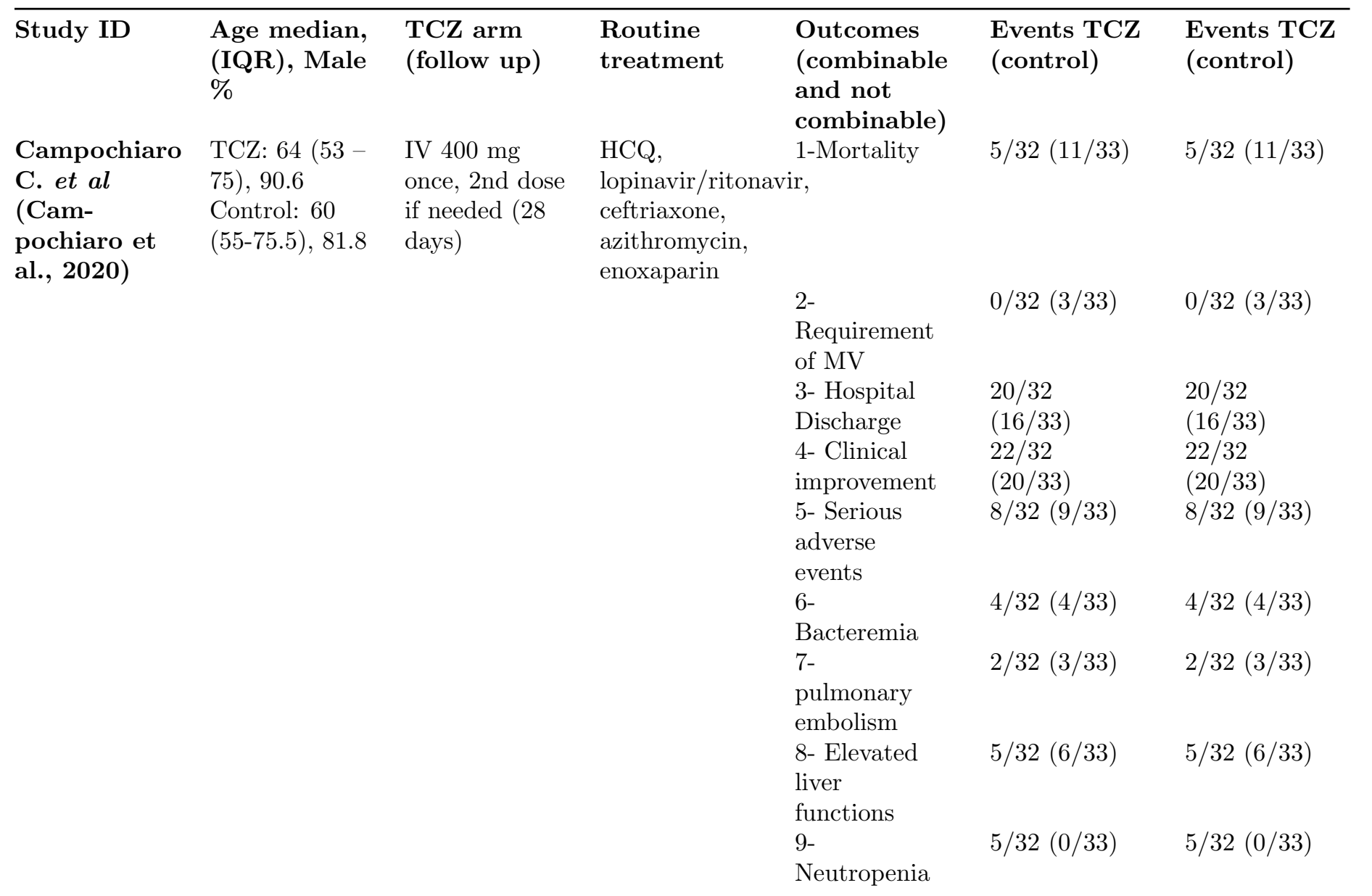




$\begin{array}{ll}\text { Guaraldi G. } & \text { TCZ: } 63 \\ \text { et al } & (54-72), 72.7 \\ \text { (Guaraldi et } & \text { Control: } 69 \\ \text { al., 2020) } & (57-78), 63.6\end{array}$

Colaneri M.

et al

(Colaneri et

al., 2020)

TCZ: 63.74

(16.32), 90.5

Control: 62.33

(18.68), 69.2

Kewan T. et TCZ: 62 (53-

al (Kewan et 71), 71.4

al., 2020)
IV $8 \mathrm{mg} / \mathrm{kh}$, Max 800, twice (14 days)

IV $8 \mathrm{mg} / \mathrm{kh}, \quad$ HCQ, Max 800, twice ( 7 days)

IV $8 \mathrm{mg} / \mathrm{kh}$, Max 400 (21 days)

HCQ,
Oxygen supply, HCQ, azithromycin, lopinavirritonavir and low molecular weight heparin

2 -

Requirement $\quad(57 / 365)$ of MV

3- Serious adverse events 5 -

Bacteremia

6- New infections 7- Elevated liver functions

$8-$

Neutropenia

1-Mortality

azithromycin, low weight heparin, and methylprednisolone Azithromycin (53 - 71), 47.8

Improved $24 / 32(20 / 33)$

Worsened 5/32

(12/33) No

change $3 / 32$

$(1 / 33)$

$6 / 88(73 / 365)$
Improved 24/32 (20/33)

Worsened $5 / 32$

$(12 / 33)$ No change $3 / 32$

$(1 / 33)$ $6 / 88(73 / 365)$
$1 / 179$

$(0 / 365)$

$3 / 179$

$(4 / 365)$

$24 / 179$

$(14 / 965)$

$0 / 179$

$(1 / 365)$

$1 / 179$

$(0 / 365)$

$5 / 21(19 / 91)$
$16 / 88$

$(57 / 365)$

$1 / 179$

$(0 / 365)$

$3 / 179$

$(4 / 365)$

$24 / 179$

$(14 / 965)$

$0 / 179$

$(1 / 365)$

$1 / 179$

$(0 / 365)$

$5 / 21(19 / 91)$
2- ICU admission

$3 / 21(12 / 91)$

$3 / 28(2 / 23)$

$3 / 21(12 / 91)$

1-Mortality

$2-$

Requirement of MV

3- Hospital Discharge

4- Clinical improvement 5- New infections
$2 / 9(6 / 18)$

$11 / 28$

$(13 / 23)$

$18 / 28$

$(13 / 23)$

$5 / 28(5 / 23)$
$2 / 9(6 / 18)$

$3 / 28(2 / 23)$

$11 / 28$

$(13 / 23)$

$18 / 28$

$(13 / 23)$

$5 / 28(5 / 23)$ 




TCZ: Tocilizumab, HCQ: hydroxychloroquine, SD: standard deviation

Table (2): Risk of bias assessment

\begin{tabular}{lllll}
\hline Tool & Campochiaro C. et al & Guaraldi G. et al & Colaneri M. et al & Kewan \\
\hline Bias due to confounding & 1 & 3 & $?$ & \\
Bias in selection of participants into the study & 1 & 1 & 1 & 1 \\
Bias in classification of interventions & 1 & 1 & 1 & 1 \\
Bias due to deviations from intended interventions & 1 & 2 & 1 & 2 \\
Bias due to missing data & 1 & 1 & 2 & 1 \\
Bias in measurement of outcomes & 2 & 2 & $?$ & $?$ \\
Bias in selection of the reported result & 1 & 1 & 2 \\
\hline
\end{tabular}

\section{Hosted file}

Fig. 1.docx available at https://authorea.com/users/360174/articles/481861-tocilizumab-intreatment-of-severe-covid-19-patients-a-systematic-review-and-meta-analysis-of-cohortstudies 


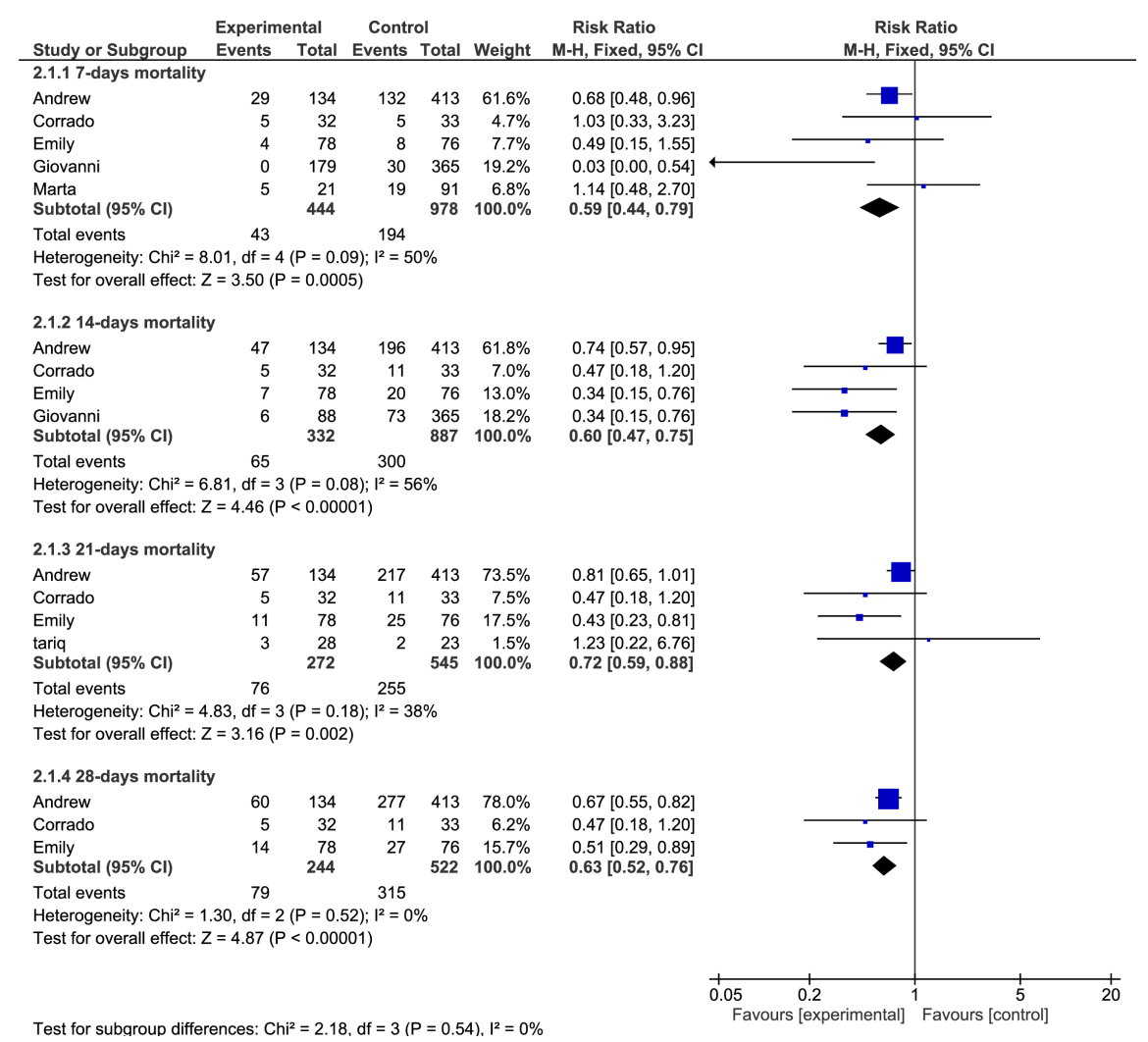

Test for subaroup differences: $\mathrm{Chi}^{2}=2.18 . \mathrm{df}=3(\mathrm{P}=0.54) . \mathrm{I}^{2}=0 \%$

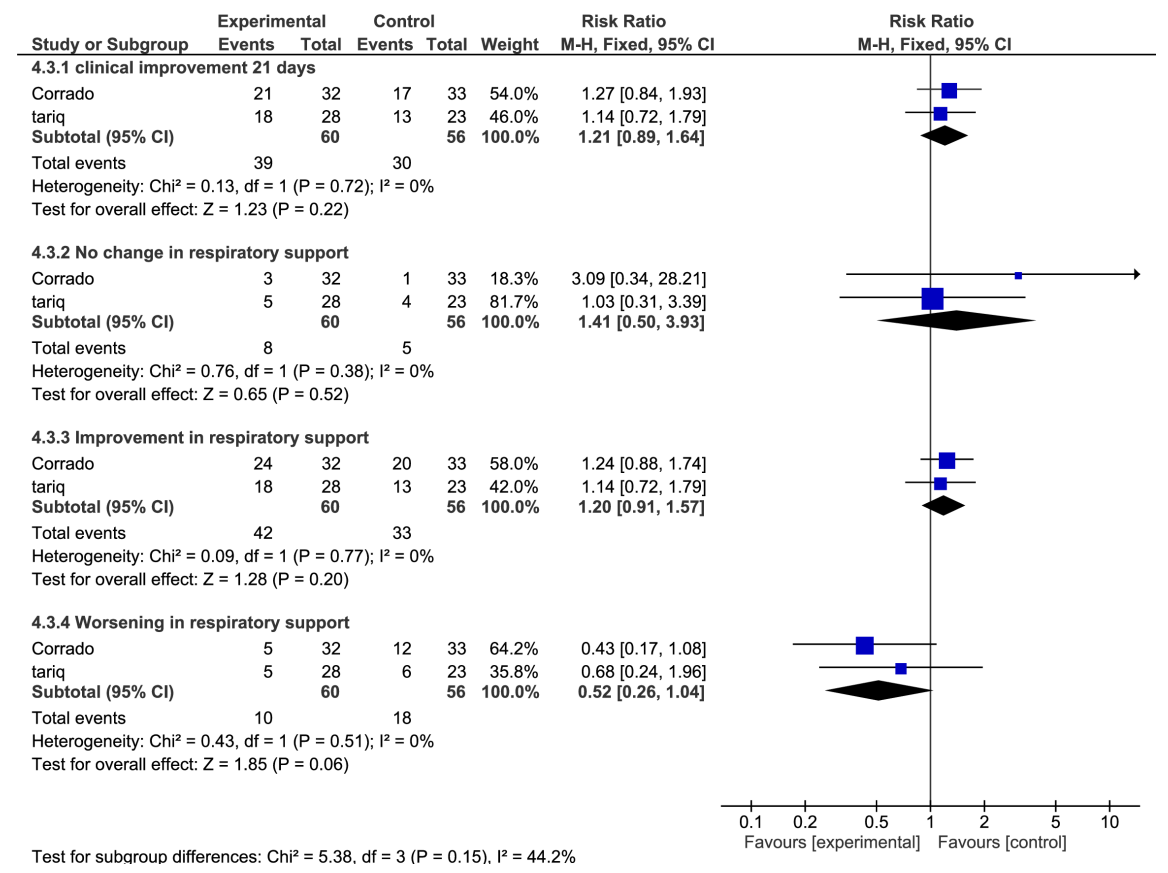




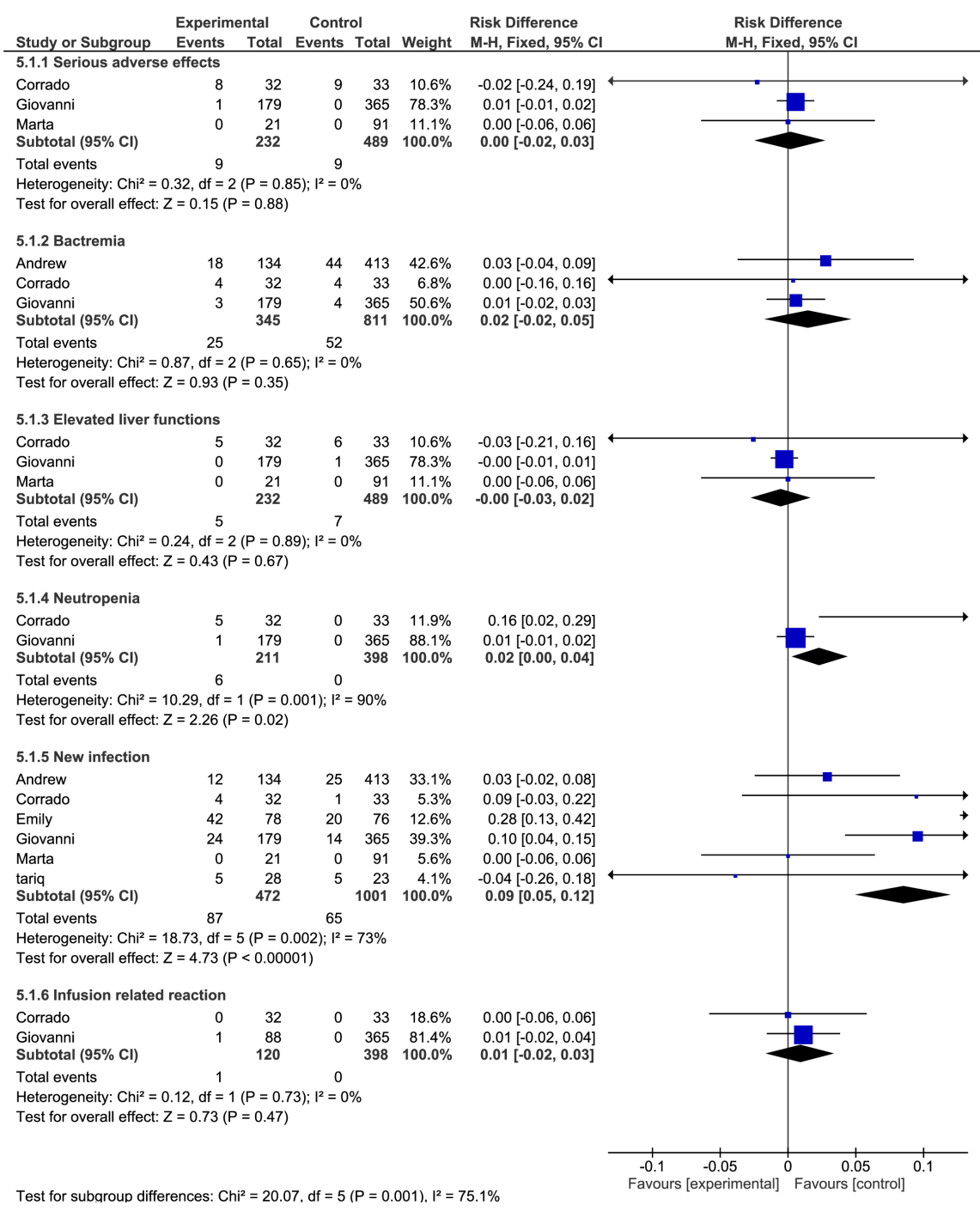

